\title{
可展收豆英杆刚度及收展过程力学分析
}

\author{
张涛涛 从强 任 晗 郭一竹 \\ (北京空间飞行器总体设计部 北京 100094)
}

\begin{abstract}
摘要: 大变形薄壁可展收复合材料结构由于其良好的力学性能和折展功能, 适用于空间探测领域。针对空间豆英杆可展收结 构, 开展了不同构型参数、不同铺层方式下的豆英杆刚度及稳定性分析。通过悬臂豆英杆的弯曲与扭转试验验证了数值模型 的正确性。采用显式非线性分析方法, 对豆英杆盘卷收拢过程进行了准静态数值模拟。结果分析表明, 豆英杆截面半径越小, 铺层厚度越厚, 收拢过程的应力水平越大; 盘卷的卷筒直径越小, 收拢过程的应力水平越大。分析结果为空间可展收豆荚杆 的设计提供了理论依据。
\end{abstract}

关键词: 纤维增强复合材料; 豆英杆; 刚度; 收展过程; 力学分析

中图分类号: TB330

\section{Folding and Stiffness Analysis for Thin-walled Deployable Boom}

\author{
ZHANG Taotao CONG Qiang REN Han GUO Yizhu
}

(Beijing Institute of Spacecraft System Engineering, Beijing 100094)

\begin{abstract}
Thin-walled deployable composite structures with high deformability are of considerable interest and increasingly used in aerospace field due to their superior mechanical behaviour and folding function. The stiffness and stability for the space thin-walled deployable composite boom are analysed with different configuration parameters and layup. The numerical model is demonstrated through experimental bending and torsion tests of cantilever boom. The quasi static folding process is numerical simulated used explicit non-linear method. Analysis results show that the boom section radius is smaller and the layup is thicker, the stress level of boom is greater during folding process. The drum diameter is smaller, the stress level of boom is greater during folding process. Analysis results provide a reference basis for thin-walled composite boom and winding institutional design.
\end{abstract}

Key words: fibre reinforced composite; thin-walled boom; stiffness; folding process; mechanical analysis

\section{0 前言}

可展收的薄壁碳纤维增强树脂基复合材料 (Carbon fibre reinforced plastics, CFRP)豆荚杆具有 收展原理简单、轻质、收纳率高、可重复性强等特 点, 可用作太阳帆、空间薄膜天线阵面等可收展的 航天器的支撑体系构件。薄壁豆荚杆展开过程为由 捆压机构施加管片挤压力, 使两凸面弹性屈曲成为 扁平管, 从而具有极小面外外刚度(仍具有高平面内 刚度)，然后绕卷筒弯曲缠绕实现收拢。

在国内，白江波等 ${ }^{[-4]}$ 对可折叠复合材料豆荚杆 的制备展开研究并进行了验证, 开展了豆荚杆的卷绕 有限元分析, 针对空间环境开展了温度对豆荚杆轴向

20200720 收到初稿, 20201207 收到修改稿
压缩的失稳分析。陈务军等 ${ }^{[5-8]}$ 对薄壁 CFRP 豆荚杆 压扁、拉扁、缠绕和展开过程进行了系统研究，并开 展了轴压屈曲特性分析和模态分析。丁峻宏等 ${ }^{[9-14]}$ 对 豆荚杆收展过程开展了并行仿真过程分析。在国外, 欧洲航天局和德国宇航中心在充气杆和弹性薄壁管展 开机构轻量化、收展机理进行了大量研究工作。德国 宇航中心研制了高性能大型透镜式薄壁 CFRP 管空间 伸展臂 ${ }^{[15]}$ 。LAURENZI 等 ${ }^{[16-17]}$ 数值模拟研究了 $\mathrm{C}$ 型截 面的薄壁管的非线性失稳和展收过程。FERNANDEZ 等 ${ }^{[18-19]}$ 分析了薄壁可展收管的结构刚度、形状和铺层方 式等的对展收性能的影响。上述文献对豆荚杆的刚度 及收展过程开展了分析, 但豆荚杆设计截面参数对刚 度及卷绕过程的影响研究尚未发现。

本文首先对豆荚杆悬臂梁进行末端加载试验, 测 试了两个方向弯曲、扭转的刚度及失稳载荷。采用有 限元软件 ABAQUS 进行建模分析, 试验结果与分析 
结果对比, 验证了分析模型的正确性。基于试验和模 拟分析, 进一步对豆荚杆壁厚、铺层层数和截面半径 进行分析, 研究了各参数对豆荚杆悬臂梁的刚度及屈 曲临界载荷的影响。最后, 采用显式非线性分析方法, 对豆荚杆盘卷收拢过程进行了准静态数值模拟, 分析 了豆荚杆截面半径、铺层厚度、卷绕筒直径参数对豆 荚杆的卷绕后应力水平的影响。分析结果为空间可展 开豆荚杆的设计提供了理论依据。

1 豆荚杆刚度及稳定性分析与试验 验证

\section{1 有限元模型}

分析模型如图 1 所示。豆荚杆长度为 $2500 \mathrm{~mm}$, 截面尺寸半径为 $50 \mathrm{~mm}$ 。复合材料铺层部分采用二 维壳单元 $\mathrm{S} 4 \mathrm{R}$, 飞边的胶层采用 C3D8R 三维单元。 胶层与铺层单元 Tie 约束。

豆荚杆采用 T300/TDE386 环氧树脂复合材料, 纤维体积含量 $50 \%$, 胶接区采用 $\mathrm{J} 159$ 胶膜, 厚度为 $0.06 \mathrm{~mm}$ 。豆荚杆 $\Omega$ 顶端为 7 层铺层 $[45 /-45 / 0 / 0 / 0$ / $-45 / 45], \Omega$ 两边为 5 层铺层 $[45 /-45 / 0 /-45 / 45], 0^{\circ}$ 方
向为杆件长度方向, 单层厚度 $0.04 \mathrm{~mm}$ 。

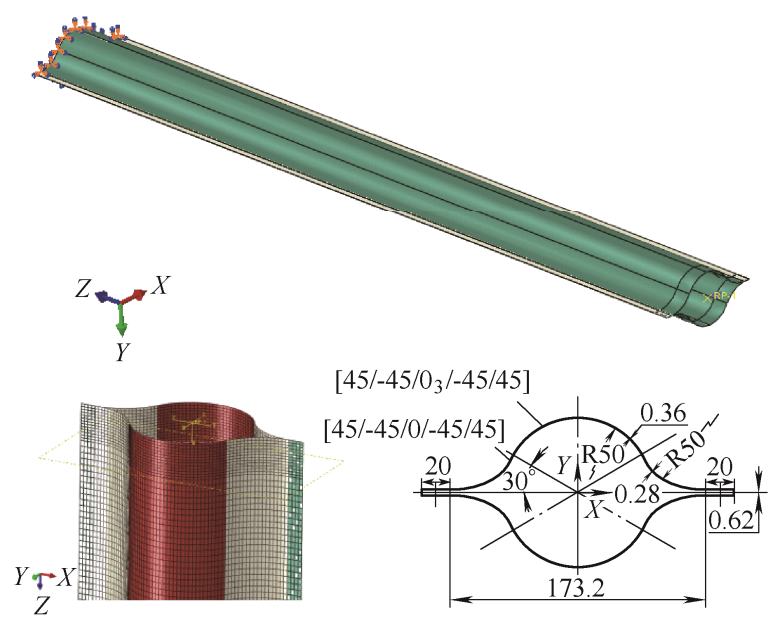

图 1 复合材料豆英杆分析模型及截面形状

采用悬臂梁加载计算方法。边界条件为一端固 支, 另一端施加两个方向集中单位弯矩载荷。

材料参数如表 1 所示。其中 $E_{11} 、 E_{22}$ 分别为 T300/TDE386 环氧树脂基复合材料单向板纵向、横向 弹性模量, $\mu_{12}$ 为面内泊松比, $G_{12} 、 G_{13} 、 G_{23}$ 分别为三 个方向切变模量, $E$ 为胶膜弹性模量, $\rho$ 为材料密度。

表 1 豆荚杆的复合材料参数(T300/TDE386 环氧树脂, 纤维体积含量 50\%)

\begin{tabular}{|c|c|c|c|c|c|c|c|}
\hline \multirow{4}{*}{$\begin{array}{c}\text { T300/TDE386 环 } \\
\text { 氧树脂 }\end{array}$} & $\begin{array}{c}\text { 纵向弹性模量 } \\
E_{11} / \mathrm{GPa} \\
\end{array}$ & $\begin{array}{c}\text { 横向弹性模量 } \\
E_{22} / \mathrm{GPa} \\
\end{array}$ & 泊松比 $\mu_{12}$ & $\begin{array}{c}\text { 切变模量 } \\
G_{12} / \mathrm{MPa} \\
\end{array}$ & $\begin{array}{c}\text { 切变模量 } \\
G_{13} / \mathrm{MPa} \\
\end{array}$ & $\begin{array}{c}\text { 切变模量 } \\
G_{23} / \mathrm{MPa} \\
\end{array}$ & $\begin{array}{c}\text { 密度 } \\
\rho /\left(\mathrm{kg} / \mathrm{m}^{3}\right) \\
\end{array}$ \\
\hline & 115 & 7 & 0.31 & 3740 & 3740 & 3500 & 1600 \\
\hline & $\begin{array}{c}\text { 纵向拉伸强度 } \\
X_{t} / \mathrm{MPa} \\
\end{array}$ & $\begin{array}{c}\text { 纵向压缩强度 } \\
X_{c} / \mathrm{MPa} \\
\end{array}$ & $\begin{array}{c}\text { 横向拉伸强度 } \\
Y_{t} / \mathrm{MPa} \\
\end{array}$ & $\begin{array}{c}\text { 横向压缩强度 } \\
Y_{c} / \mathrm{MPa} \\
\end{array}$ & $\begin{array}{c}\text { 剪切强度 } \\
S / \mathrm{MPa} \\
\end{array}$ & & \\
\hline & 1833 & 1027 & 117 & 140 & 151 & & \\
\hline \multirow[t]{2}{*}{$\mathrm{J} 159$ 胶膜 } & 弹性模量 $E / \mathrm{GPa}$ & $\begin{array}{c}\text { 密度 } \\
\rho /\left(\mathrm{kg} / \mathrm{m}^{3}\right) \\
\end{array}$ & & & & & \\
\hline & 5 & 1600 & & & & & \\
\hline
\end{tabular}

\section{2 分析结果}

在豆荚杆端部施加单位弯矩载荷 $M$, 提取端部 转角 $\theta$, 计算刚度参数如下

$$
\begin{aligned}
& (E I)_{x}=M_{x} l / \theta_{x} \\
& (E I)_{y}=M_{y} l / \theta_{y}
\end{aligned}
$$

式中, $E I$ 是弯曲刚度, $l$ 为豆荚杆长度。

同时, 计算了一端固支, 另一端自由施加轴向 压载荷的整体失稳和局部屈曲载荷。
表 2 为复合材料豆荚杆的刚度分析结果。其中, $(E I)_{x} 、(E I)_{y}$ 分别为 $x 、 y$ 向弯曲刚度; 7/5Ply 表示豆 荚杆 $\Omega$ 顶弧为 7 层铺层 $[45 /-45 / 0 / 0 / 0 /-45 / 45], \Omega$ 两 边侧弧为 5 层铺层 $[45 /-45 / 0 /-45 / 45] ; 8 / 6 \mathrm{Ply}$ 表示豆 荚杆 $\Omega$ 顶弧为 8 层铺层 $[45 /-45 / 0 / 0 / 0 / 0 /-45 / 45], \Omega$ 两边侧弧为 6 层铺层 $[45 /-45 / 0 / 0 /-45 / 45]$; 9/7Ply 表 示豆荚杆 $\Omega$ 顶弧为 9 层铺层 $[45 /-45 / 0 / 0 / 0 / 0 / 0 /-45 /$ $45], \Omega$ 两边侧弧为 7 层铺层 $[45 /-45 / 0 / 0 / 0 /-45 / 45]$ 。

\begin{tabular}{|c|c|c|c|c|c|c|}
\hline \multirow{2}{*}{ (计算分析模型为豆荚杆长度 $2500 \mathrm{~mm}$ ) } & \multicolumn{3}{|c|}{ 豆荚杆截面半径 $50 \mathrm{~mm}$} & \multicolumn{3}{|c|}{ 豆荚杆截面半径 $80 \mathrm{~mm}$} \\
\hline & 7/5Ply & 8/6Ply & 9/7Ply & 7/5Ply & 8/6Ply & 9/7Ply \\
\hline$x$ 向弯曲刚度 $(E I)_{x} /\left(10^{4} \mathrm{~N} \cdot \mathrm{m}^{2}\right)$ & 0.66 & 0.84 & 1.03 & 2.70 & 3.46 & 4.23 \\
\hline$y$ 向弯曲刚度 $(E I)_{y} /\left(10^{4} \mathrm{~N} \cdot \mathrm{m}^{2}\right)$ & 1.47 & 2.29 & 3.12 & 5.11 & 7.89 & 10.64 \\
\hline 整体失稳载荷 $/ \mathrm{kN}$ & 2.59 & 3.31 & 4.05 & - & - & - \\
\hline 局部屈曲载荷/kN & 3.29 & 5.06 & 7.0 & 3.38 & 5.17 & 7.14 \\
\hline
\end{tabular}

表 2 复合材料豆荚杆刚度分析结果(端部施加单位弯曲载荷) 
从表 2 中可以看出: 豆荚杆的 $y$ 向弯曲刚度是 $x$ 向刚度的 2 3 倍; 增加 $0^{\circ}$ 铺层可以明显增加弯 曲刚度。 $2500 \mathrm{~mm}$ 长的豆荚杆截面半径为 $50 \mathrm{~mm}$ 时, 失稳首先为整体失稳; 半径为 $80 \mathrm{~mm}$ 时, 首先 出现局部失稳。

\section{3 试验验证}

试验件形状及截面尺寸如图 1 所示。半径为 $50 \mathrm{~mm}$, 长度为 $1500 \mathrm{~mm}$ 的豆英杆试验件, 铺层状 态为 9/7Ply: 其中豆荚杆 $\Omega$ 顶弧为 9 层铺层 $[45 /-45 / 0 / 0 / 0 / 0 / 0 /-45 / 45], \Omega$ 两边侧弧为 7 层铺层 $[45 /-45 / 0 / 0 / 0 /-45 / 45], 0^{\circ}$ 方向为杆件轴向。

试验件共 3 件, 测试刚度、局部屈曲。将豆荚 杆两端封堵, 防止边缘局部变形。堵头与试验加载 端工装及固定端工装一体化设计。两端堵头分别由 内腔盖、2 个外包半环、 1 个加载端或固定端盖板组 成。内腔盖、外包环的截面与豆荚杆侧壁贴合, 缝 隙不超过 $0.1 \mathrm{~mm}$ 。粘接过程需在贴合面两侧涂抹均 匀胶。

试验工况分别为: (1) 豆荚杆一端固定, 另一端 逐级施加 $x$ 向的横向载荷测试弯曲刚度。横向载荷 最大施加 $50 \mathrm{~N}$, 等分 5 级逐级施加; (2) 豆荚杆一 端固定, 另一端逐级施加 $y$ 向的横向载荷测试弯曲 刚度。横向载荷最大施加 $50 \mathrm{~N}$, 等分 5 级逐级施加; (3) 将豆荚杆一端固定, 另一端逐级施加扭矩测试扭 转刚度。扭矩最大施加 $30 \mathrm{~N} \cdot \mathrm{m}$, 等分 5 级逐级施加。 最后, 开展 $y$ 向载荷失稳测试和扭转载荷失稳测试。

测试的刚度曲线如图 2 所示, 图中纵坐标为悬 臂杆加载端中心点的位移和转角值。从图中可以看 出, 加载与卸载曲线近似重合, 表明试验测试过程 与结果正确。

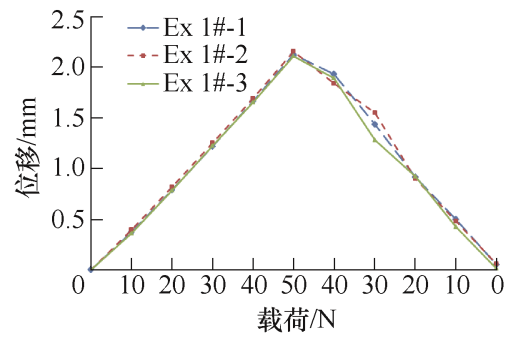

(a) $x$ 向弯曲刚度载荷-位移曲线

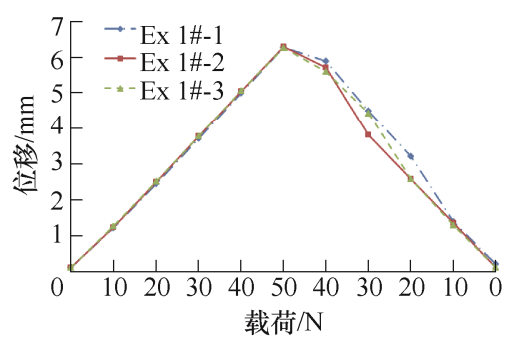

(b) $y$ 向弯曲刚度载荷-位移曲线

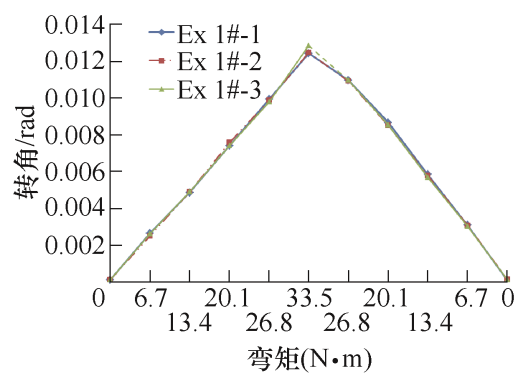

(c) 扭转刚度载荷-转角曲线

图 2 试验载荷-位移/转角曲线

表 3 为测试载荷与分析值对比结果。从表中可以 看出, 弯曲刚度与扭转刚度的测试值与分析值吻合较 好, 误差在 $15 \%$ 以内, 失稳载荷的试验值约为分析值 的 $60 \%$, 说明薄壁豆荚杆在失稳系数选择的过程中需 要有较大的安全裕度。图 3a、3b 分别为 $y$ 向失稳模态 与扭转失稳模态的有限元模拟与试验结果。从图中可 以看出, 分析与试验的失稳模态吻合良好。

表 3 复合材料豆荚杆试验与分析对比

\begin{tabular}{|c|c|c|c|}
\hline & \multicolumn{3}{|c|}{$\begin{array}{c}\text { 豆荚杆截面半径 R50, 长度 } \\
1500 \mathrm{~mm}, 9 / 7 \text { 铺层 } \\
\end{array}$} \\
\hline & 试验 & 分析 & 误差(\%) \\
\hline$x$ 向弯曲刚度/( N/mm) & 23.5 & 27 & 13 \\
\hline$y$ 向弯曲刚度/( N/mm) & 9.3 & 10.1 & 8 \\
\hline 扭转刚度/( $\mathrm{N} \cdot \mathrm{m} / \mathrm{mrad})$ & 2.11 & 2.4 & 13 \\
\hline$y$ 向局部失稳载荷/ N & 150 & 235 & \\
\hline 扭转失稳载荷/( $\mathrm{N} \cdot \mathrm{m})$ & 40 & 67 & \\
\hline
\end{tabular}

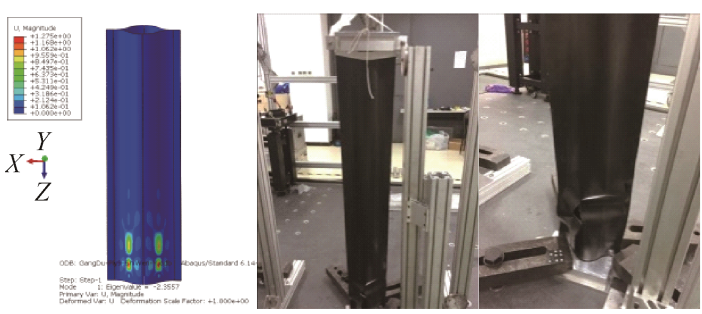

(a) $y$ 向弯曲局部失稳模态分析与试验对比

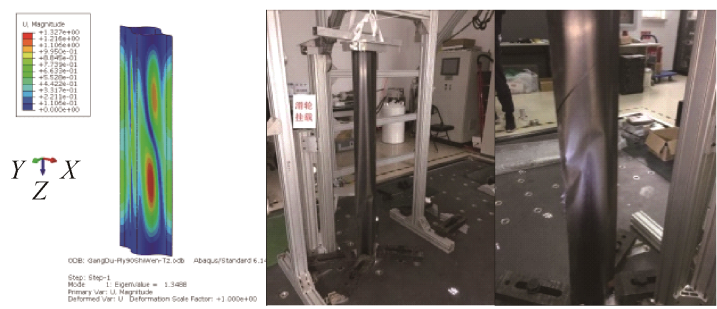

(b) 轴向扭转局部失稳模态分析与试验对比

图 3 失稳模态分析与试验对比

2 豆荚杆收展过程分析

\section{1 有限元模型}

取半径为 $80 \mathrm{~mm}$, 三种不同铺层形式的豆荚杆 
建立有限元模型, 模型如图 4 所示。由卷筒、12 个 约束转轴和豆荚杆组成, 相对位置关系如图 4 右下 所示。由于受力和结构的对称性, 采用 $1 / 2$ 模型进 行分析。

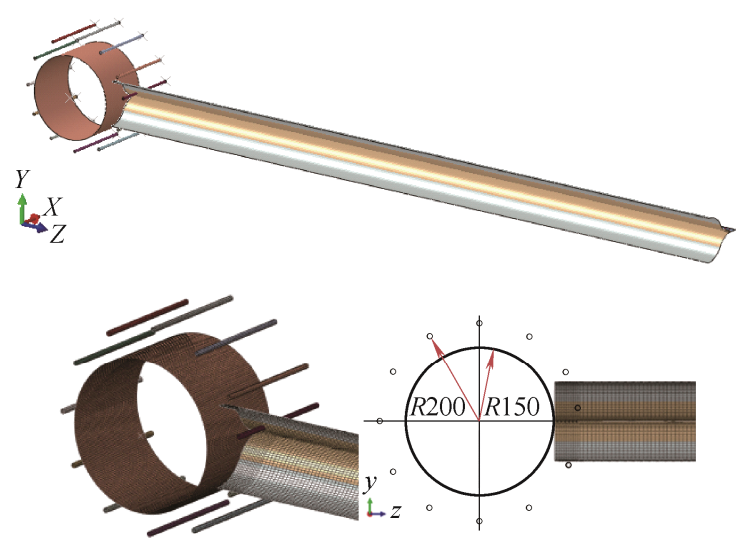

图 4 复合材料豆英杆折叠有限元模型

其中, 卷筒、约束转轴为刚性单元 R3D4, 圆 心位置建立参考点, 刚性单元随参考点运动。豆荚 杆为壳单元 $S 4 R$, 胶层单元为 C3D8R 单元, 豆英杆 飞边与胶层单元上下表面粘接约束。卷筒单元数 11800 个, 每个约束转轴单元数 512 个, 豆荚杆单 元数 24244 个, 胶层单元数 3200 个。

\section{2 边界条件}

复合材料豆荚杆边界约束如图 5 所示。在复合 材料豆荚杆轴对称位置施加对称边界条件, 将端部 飞边的棱边与卷筒的随动点设置铰链约束。卷筒转 轴通过参考点运动, 约束转轴通过参考点约束六个 自由度。

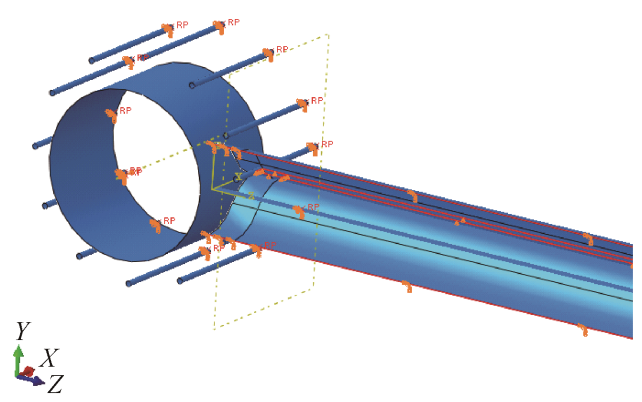

图 5 豆荚杆折叠过程边界条件

卷筒分两步作动: 第一步将豆荚杆拉扁, 此时 卷筒的参考点除了沿转动轴的平移自由度外, 其他 自由度全部约束; 第二步通过转动将豆英杆收拢, 此时其参考点除了沿转动轴的转动自由度外, 其他 自由度全部约束。

卷筒、约束转轴和豆荚杆彼此之间的接触采用 通用接触方式, 接触属性发向为硬接触, 切向为摩
擦接触, 摩擦系数取 0.05 。

\section{3 分析结果}

(1) 位移分析。

豆英杆拉扁后的位移云图如图 6 所示, 从图中 看出在拉扁到 $29.1 \mathrm{~mm}$ (理论拉扁尺寸为 $29 \mathrm{~mm}$ ) 后, 豆荚杆端部的自由端张口边缘接近重合。

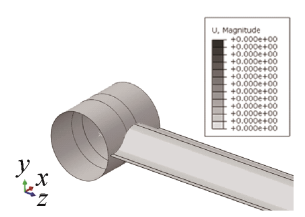

(a) 初始状态

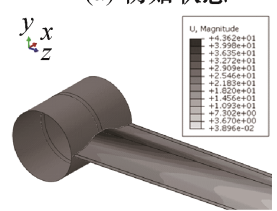

(c) 拉扁 $23.3 \mathrm{~mm}$

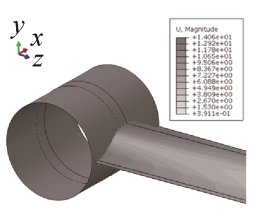

(b) 拉扁 $11.6 \mathrm{~mm}$ (d) 拉.扁 $29.1 \mathrm{~mm}$

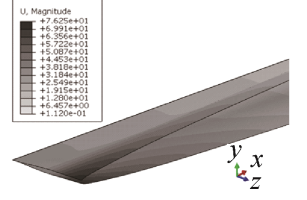

(e) 拉扁 $29.1 \mathrm{~mm}$ (局部变形)

图 6 豆荚杆拉扁过程位移云图(单位 $\mathrm{mm}$ )

豆荚杆卷曲位移云图如图 7 所示, 图中分别为 卷曲 1.0 和 2.0 圈的变形云图。

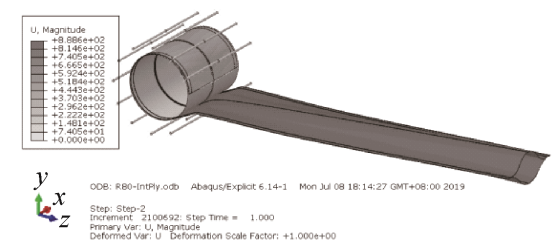

(a) 卷曲 1.0 圈

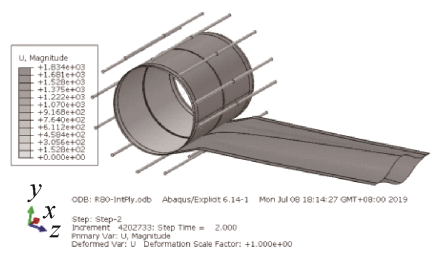

(b) 卷曲 2.0 圈

图 7 豆荚杆卷曲过程位移云图(单位 $\mathrm{mm}$ )

(2) 应力分析。

豆荚杆卷曲过程的应力水平分为三个位置开展 分析, 分别为紧贴卷筒位置, 端部靠近飞边位置, 约束转轴折弯位置, 如图 8 所示。以卷曲 0.9 圈为 例分析, 其他卷曲过程与此类似。

其中, 位置 2 为应力集中区域, 最大应力 551.8 $\mathrm{MPa}$, 但此位置由于实际状态为在端部施加均匀线 载荷, 而分析状态为了便于计算收玫以及施加边界 约束条件, 在端部施加集中点载荷, 故分析状态较 实际状态值偏大, 因此忽略。位置 3 为约束转轴折 
弯位置, 最大应力 $361.3 \mathrm{MPa}$, 此部分的最大应力 与折弯曲率有关, 需考虑具体工程中的实际情况。

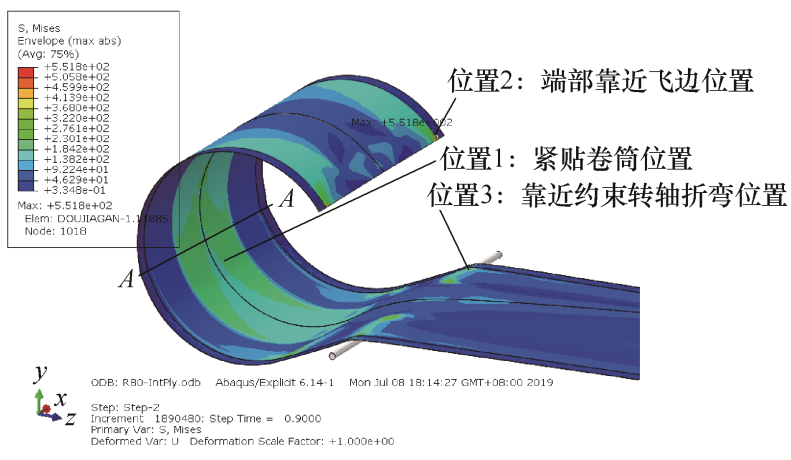

图 8 豆荚杆卷曲过程中应力云图( $\mathrm{MPa})$

重点考虑紧贴卷筒位置 1 的应力水平, 其中, $7 / 5$ 铺层状态的应力水平约为 $200 \mathrm{MPa} ; 8 / 6$ 铺层状 态的应力水平约为 $225 \mathrm{MPa}$; $9 / 7$ 铺层状态的应力水 平约为 $250 \mathrm{MPa}$ 。说明在卷筒曲率一定的情况下, 正应力与厚度成正比。采用 ABAQUS 中的 Tsai-Wu 准则校核豆荚杆的失效系数。 Tsai-Wu 失效准则为 $I_{F}=F_{1} \sigma_{11}+F_{2} \sigma_{22}+F_{11} \sigma_{11}^{2}+F_{22} \sigma_{22}^{2}+F_{12} \sigma_{12}^{2}+2 F_{12} \sigma_{11} \sigma_{22}<$ 1.0 , 其中 $F_{1}=1 / X_{\mathrm{t}}+1 / X_{\mathrm{c}}, F_{2}=1 / Y_{\mathrm{t}}+1 / Y_{\mathrm{c}}, F_{11}=-1 / X_{\mathrm{t}} X_{\mathrm{c}}$, $F_{22}=-1 / Y_{\mathrm{t}} Y_{\mathrm{c}}, F_{66}=1 / S^{2}$, 其中 $\sigma_{i j}$ 为各方向应力值, $X_{\mathrm{t}}$ 、 $X_{\mathrm{c}} 、 Y_{\mathrm{t}} 、 Y_{\mathrm{c}} 、 S$ 为强度值见表 1 所示。失效系数分别 为 $0.226,0.256,0.286$ 。

另外, 提取沿图 8 中 $A-A$ 截面的紧贴卷筒壁面 的豆荚杆内圈和外圈的 $\sigma_{11}$ 应力分布, 如图 9 所示。 从图中看出, 在外圈中间 $(\Omega$ 弧顶 $) 7$ 层铺层中, 纤维 受拉为主, 量级不超过 $100 \mathrm{MPa}$; 在内圈中间 $(\Omega$ 弧 顶)7 层铺层中, 上表面受拉, 下表面受压, 上下表 面接近对称。在外圈边缘 $(\Omega$ 两边 $) 5$ 层铺层中, 内外 表面受拉为主, 纤维方向最大拉应力约 $150 \mathrm{MPa}$, 纤维方向最大压应力约 $-50 \mathrm{MPa}$; 内圈边缘 $(\Omega$ 两 边) 5 层铺层中, 内外表面应力水平不大。

应力水平采用弯曲梁理论公式估算, 符合实际 情况。在外表面受拉应力水平下, 外表面的伸长量 大于内表面, 增量与内外表面的周长差在一个数量
级, 内外表面的周长差通过变形抵消, 未经过飞边 部分传递载荷, 在一定程度可以说明在飞边处剪切 应力较低的原因。

此外, 分析了豆荚杆在半径 $50 \mathrm{~mm}$ 和 $80 \mathrm{~mm}$ 情况下的不同铺层下的强度裕度, 见表 4 所示。 表 5 汇总了豆荚杆半径 $80 \mathrm{~mm}, 7 / 5 \mathrm{Ply}$ 铺层方式在 不同卷筒直径下的强度裕度。从表 4 和表 5 看出在 卷曲过程中, 在卷筒曲率、豆荚杆半径相同的情况 下, 紧贴卷筒壁面的应力水平与豆荚杆铺层厚度成 正比; 在卷筒曲率、豆荚杆厚度一定的情况下, 半 径为 $50 \mathrm{~mm}$ 的豆荚杆强度裕度要低于半径为 $80 \mathrm{~mm}$ 的豆荚杆; 在豆荚杆铺层、直径一定的情况下, 卷 筒直径越小, 强度裕度越低。

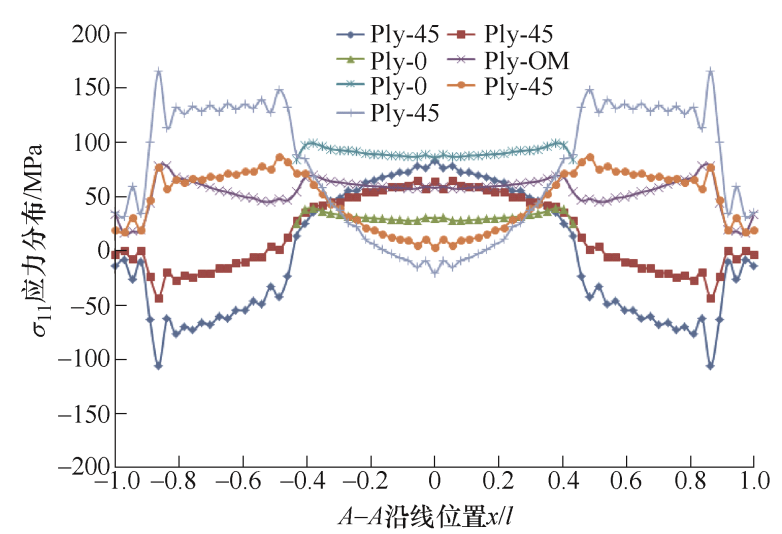

(a) 内圈

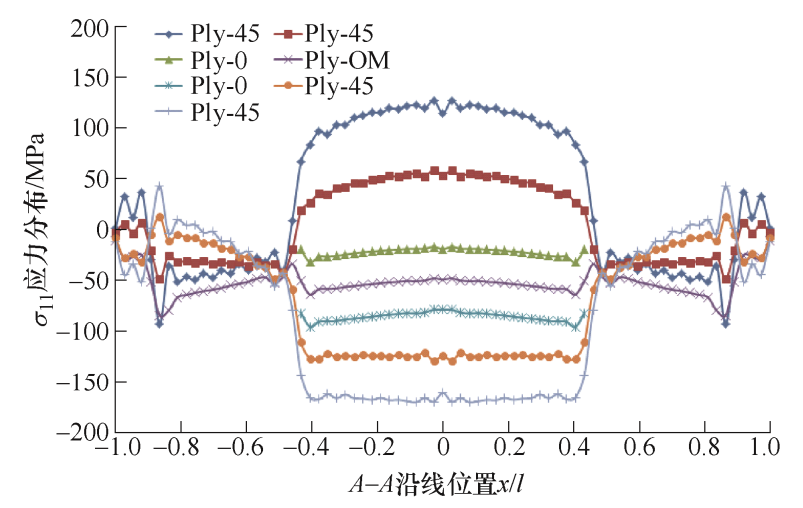

(b) 外圈

图 9 豆荚杆卷曲 0.9 圈时在 $A-A$ 截面上的应力分布情况

表 4 豆荚杆在卷筒直径 $300 \mathrm{~mm}$ 时的强度裕度

\begin{tabular}{|c|c|c|c|c|c|c|}
\hline & & \multicolumn{2}{|c|}{ 豆荚杆半径 $50 \mathrm{~mm}$} & \multicolumn{3}{|c|}{ 豆荚杆半径 $80 \mathrm{~mm}$} \\
\hline & & 7/5Ply & 9/7Ply & 7/5Ply & 8/6Ply & 9/7Ply \\
\hline \multirow{2}{*}{ 胶层应力水平 $/ \mathrm{MPa}$} & 剥离 & 3.606 & 2.885 & 2.65 & 2.99 & 4.066 \\
\hline & 剪切 & 2.591 & 1.185 & 3.01 & 2.83 & 3.594 \\
\hline \multirow{3}{*}{$\begin{array}{c}\text { 卷曲位置 } \\
1\end{array}$} & 铺层应力水平 $/ \mathrm{MPa}$ & 258.3 & 308.7 & 197.7 & 224.1 & 250.6 \\
\hline & 铺层失效系数 $R$ & 0.303 & 0.375 & 0.226 & 0.256 & 0.286 \\
\hline & 强度裕度 $1 /(R-1)$ & 2.3 & 1.67 & 3.42 & 2.91 & 2.5 \\
\hline \multirow{3}{*}{$\begin{array}{c}\text { 卷曲位置 } \\
3\end{array}$} & 应力水平/MPa & 322.2 & 480.9 & 361.3 & 448.2 & 529.3 \\
\hline & 失效系数 $R$ & 0.387 & 0.55 & 0.287 & 0.355 & 0.445 \\
\hline & 强度裕度 $1 /(R-1)$ & 1.58 & 0.82 & 2.48 & 1.82 & 1.25 \\
\hline
\end{tabular}


表 5 豆荚杆半径 $80 \mathrm{~mm}, 7 / 5 \mathrm{Ply}$ 在不同卷筒直径下的强度裕度

\begin{tabular}{|c|c|c|c|c|c|}
\hline & 直径 $/ \mathrm{mm}$ & 300 & 250 & 200 & 150 \\
\hline \multirow{3}{*}{ 卷曲位置 1} & 应力水平 $/ \mathrm{MPa}$ & 197.7 & 215.8 & 227.5 & 243.6 \\
\hline & 失效系数 $R$ & 0.226 & 0.236 & 0.252 & 0.276 \\
\hline & 强度裕度 $1 /(R-1)$ & 3.42 & 3.24 & 2.97 & 2.62 \\
\hline \multirow{3}{*}{ 卷曲位置 3} & 应力水平/MPa & 361.3 & 405.9 & 415.6 & 351.1 \\
\hline & 失效系数 $R$ & 0.287 & 0.305 & 0.321 & 0.339 \\
\hline & 强度裕度 $1 /(R-1)$ & 2.48 & 2.28 & 2.11 & 1.95 \\
\hline
\end{tabular}

\section{3 结论}

分析了豆荚杆在完全展开状态下的刚度、失稳 载荷以及卷曲过程, 得出了以下结论。

(1) 豆荚杆在完全展开状态下, $y$ 向弯曲刚度是 $x$ 向刚度的 2 3 倍; 增加 $0^{\circ}$ 铺层可以明显增加弯 曲刚度; 试验值与分析值在弯曲和扭转刚度值及失 稳模态吻合较好。

(2) 豆荚杆在卷曲过程中, 在卷筒曲率、豆荚 杆半径相同的情况下, 紧贴卷筒壁面的应力水平与 豆荚杆铺层厚度成正比; 在卷筒曲率、豆荚杆厚度 一定的情况下, 半径为 $50 \mathrm{~mm}$ 的豆荚杆强度裕度要 低于半径为 $80 \mathrm{~mm}$ 的豆荚杆; 在豆荚杆铺层、直径 一定的情况下, 卷筒直径越小, 强度裕度越低。

\section{参 考 文 献}

[1] 白江波, 熊峻江, 高军鹏, 等. 可折叠复合材料豆英杆 的制备与验证[J]. 航空学报, 2011，32(7): 1217-1223. BAI Jiangbo, XIONG Junjiang, GAO Junpeng, et al. Fabrication and validation of collapsible composite lenticular tubes[J]. Acta Aeronautica et Astronautica Sinica, 2011, 32(7): 1217-1223.

[2] 林秋红, 白江波, 从强. 超长可折叠复合材料豆英杆轴 向压缩屈曲性能测定方法 [J]. 航空制造技术，2019， 62(4): $51-55$

LIN Qiuhong, BAI Jiangbo, CONG Qiang. Novel experimental method for determining axial compression buckling behaviour of ultra-length foldable thin-walled lenticular composite tube[J]. Composites Structure and Manufacturing, 2019, 62(4): 51-55.

[3] BAI Jiangbo, CHEN Di, XIONG Junjiang, et al. Folding analysis for thin-walled deployable composite boom[J]. Acta Astronautica, 2019, 159: 622-636.

[4] BAI Jiangbo, XIONG Junjiang. Temperature effect on buckling properties of ultra-thin-walled lenticular collapsible composite tube subjected to axial
compression[J]. Chinese Journal of Aeronautics, 2014, 27(5): 1312-1317.

[5] 李瑞雄, 陈务军, 付功义, 等. 透镜式缠绕肋压扁缠绕 过程数值模拟分析[J]. 宇航学报, 2011, 32(1): 224-231. LI Ruixiong, CHEN Wujun, FU Gongyi, et al. Numerical simulation of flattening and wrapping process of lenticular wrapped-rib[J]. Journal of Astronautics, 2011, 32(1): 224-231.

[6] 邹涛, 陈务军, 彭福军, 等. 透镜式缠绕肋收展过程数 值模拟分析 $[\mathrm{J}]$. 哈尔滨工程大学学报, 2013，34(2): 151-156

ZOU Tao, CHEN Wujun, PENG Fujun, et al. Numerical simulation of stowing and deploying process of lenticular wrapped-rib[J]. Journal of Harbin Engineering University, 2013, 34(2): 151-156.

[7] 李瑞雄, 陈务军, 付功义. 透镜式薄壁 CFRP 管空间伸 展臂轴压屈曲分析及试验[J]. 宇航学报, 2012, 33(8): 1164-1170.

LI Ruixiong, CHEN Wujun, Fu Gongyi. Buckling Analysis and experiment of lenticular CFRP thin-walled tube space boom under axial compression[J]. Journal of Astronautics, 2012, 33(8): 1164-1170.

[8] 高冀峰, 房光强, 余兵, 等. 织物增强形状记忆复合材 料大型空间可展开桁架研究进展 $[\mathrm{J}]$. 机械工程学报, 2020，56(5): 80-91.

GAO Jifeng, FANG Guangqiang, YU Bing, et al. Recent progress on weave-reinforced-shaped-memory-composite based space deployable turss[J]. Journal of Mechanical Engineering, 2020, 56(5): 80-91.

[9] 鞠博文, 齐俊伟, 李涛, 等. 针对半豆英杆的先进拉挤 成型压力研究 [J]. 航空材料学报, 2015, 35(6): 20-26. JU Bowen, QI Junwei, LI Tao, et al. Research on molding pressure of advanced pultrusion for half lenticular tube $[\mathrm{J}]$. Journal of Aeronautical Materials，2015， 35(5): 20-26.

[10] 肖健, 齐俊伟, 肖军, 等. 面向半豆荚杆的先进拉挤装 备技术研究 $[\mathrm{J}]$. 玻璃钢/复合材料, 2014，11：42-47. XIAO Jian, QI Junwei, Xiao Jun, et al. Research on advanced pultrusion equipment technology of half 
lenticular tube[J]. Fiber Reinforced Plastics/Composites, 2014, 11: 42-47.

[11] 杨兴瑞. 复合材料半豆荚杆动模先进拉挤技术研究[D]. 南京: 南京航空航天大学, 2014.

YANG Xingrui. Research on Moving Mold advanced pultrusion technology of composite half lenticular tube[D]. Oranjing : Nanjing University of Aeronautics and Astronautics, 2014.

[12] 周黄鹤, 齐俊伟, 诸静, 等. 半豆荚杆先进拉挤预处理 工艺研究[J]. 玻璃钢/复合材料，2015(8): 66-70.

ZHOU Huanghe, QI Junwei, CHU Jing, et al. Study on the pretreatment process of advanced pultrusion for half lenticular tube[J]. Fiber Reinforced Plastics/Composites, 2015(8): 66-70.

[13] 杨兴瑞, 肖健, 齐俊伟, 等. 面向半豆英杆的先进拉挤 新方法研究[J]. 玻璃钢/复合材料, 2014，6: 62-65.

YANG Xingrui, XIAO Jian, QI Junwei, et al. Research of a new method about the half composite lenticular tube advanced pultrusion[J]. Fiber Reinforced Plastics/ Composites, 2014, 6: 62-65.

[14] 鞠博文. 针对半豆荚杆的先进拉挤系统优化与关键技 术研究[D]. 南京: 南京航空航天大学, 2016.

JU Bowen. Research on the optimization design and key technologies of advanced pultrusion system for half lenticular tube[D]. Nanjing: Nanjing University of Aeronautics and Astronautics, 2014.

[15] NAKAMURA K, TSUTSUMI Y, UCHIMARU K, et al. Large deployable reflector on ETS-VIII[C]// Int. 17th Communications Satellite Systems Conference and Exhibit, Yokohama, Japan, Feb. 23-27, 1998: 1-10.

[16] STABILE A, LAURENZI S. Coiling dynamic analysis of thin-walled composite deployable boom[J]. Composite Structrures, 2014(113): 429-436.

[17] BARVERA D, LAURENZI S. Nonlinear buckling and folding analysis of a storable tubular ultrathin boom for nanosatellites[J]. Composite Structures, 2015(159): 226-238.

[18] FERNANDEZ JM. Advanced deployable shell-based composite booms for small satellite applications including solar sails[C]// 4th International Symposium on Solar Sailing, Kyoto, Japan, 17-20 January, 2017: 1.

[19] FERNANDEZ JM, VISAGIE L, SCHENK M, et al. Design and development of a gossamer sail system for deorbiting in low earth orbit[J]. Acta Astronaut, 2014(103): 204-225.

作者简介: 张涛涛(通信作者), 男, 1989 年出生, 博士研究生。主要研 究方向为航天器结构设计。

E-mail: zhangtaotao@buaa.edu.cn@buaa.edu.cn 